\title{
Epidemiologia dos transtornos alimentares: estado atual e desenvolvimentos futuros
} Epidemiology of eating disorders: current status and future developments

Phillipa J Hay

\author{
Departamento de Psiquiatria da Universidade de Adelaide. Austrália do Sul, Austrália
}

\begin{abstract}
Resumo Os objetivos do presente trabalho foram: fornecer uma avaliação do progresso da epidemiologia na área de transtornos alimentares, desde estudos sobre incidência e prevalência até os estudos comunitários prospectivos, bem como estudos caso-controle; sintetizar o atual estado da incidência e prevalência dos transtornos alimentares; discutir os estudos epidemiológicos analíticos sobre os transtornos alimentares, enfocando estudos comunitários sobre fatores de risco e nosologia; e apontar as áreas de estudos futuros, especialmente sobre a carga social e econômica e o grau de "conhecimento sobre saúde mental" da população em geral a respeito das pessoas com transtornos alimentares. Apesar dos problemas para identificar e recrutar um número suficiente de pessoas com anorexia nervosa e os métodos variáveis de recrutamento de casos, os estudos sobre incidência e prevalência dos transtornos alimentares atingiram um consenso e em geral não corroboram uma incidência ascendente atual, exceto, possivelmente, por um pequeno aumento na anorexia nervosa em mulheres jovens. A aplicação de métodos epidemiológicos analíticos permitiu uma compreensão melhor dos fatores ambientais e genéticos, em comparação com os sociais e econômicos, quanto ao risco de desenvolvimento de transtornos alimentares, bem como ajudaram no refinamento da nosologia desses transtornos. Futuramente, a epidemiologia analítica terá potencial para responder a questões-chave sobre a natureza e os determinantes dos transtornos alimentares e para ajudar a decidir como auxiliar os que mais necessitem.
\end{abstract}

Descritores Transtornos alimentares. Bulimia nervosa. Anorexia nervosa. Epidemiologia. Fatores de risco. Estudos longitudinais. Estudos comunitários de classificação.

Abstract The present paper aims were: to provide an overview of development of epidemiology in the area of eating disorders, from studies of incidence and prevalence, through to community based prospective as well as case controlled studies; to summarise the current status of incidence and prevalence of eating disorders; to discuss analytic epidemiological studies of eating disorders, with a focus on community-based studies of risk factors and nosology; and to point to future areas for study, notably the social and economic burden and general population "mental health literacy" of people with eating disorders. In spite of problems in identifying and recruiting sufficient numbers of people with anorexia nervosa and variable methods of case-ascertainment, studies of incidence and prevalence of eating disorders have reached a general consensus and do not in general support a current rising incidence, except possibly a small increase in anorexia nervosa in young women. The application of analytic epidemiologic methods has lead to a greater understanding of environmental and genetic factors, as compared with social and economic factors, to the risk of developing eating disorders, and as well aided the refinement of eating disorder nosology. In the future analytic epidemiology has the potential to answer key questions about the nature and determinants of eating disorders, and to help to decide how to help those who need it most.

Keywords Eating disorders. Bulimia nervosa. Anorexia nervosa. Epidemiology. Risk factors. Longitudinal studies. Classification community-based studies.

Este trabalho foi apresentado parcialmente na Reunião Científica Anual da Sociedade de Pesquisa de Transtornos Alimentares em novembro de 2001 no Novo México. 


\section{Introdução}

\section{Estudos sobre incidência e prevalência}

As estimativas sobre a prevalência dos transtornos psiquiátricos baseiam-se no reconhecimento e delineamento precisos dos transtornos nos esquemas de classificação e no desenvolvimento de métodos para os estudos epidemiológicos nas comunidades, tais como o estudo Epidemiologic Catchment Areas (ECA) em 1980. ${ }^{1}$ Embora a anorexia nervosa tenha sido o primeiro transtorno alimentar a ser reconhecido a partir dos relatórios de Gull $^{2}$ e Laségue $^{3}$ no século XIX, as síndromes bulimia nervosa, transtorno de compulsão alimentar periódica e o Transtorno Alimentar Sem Outra Especificação (TASOE) só foram descritas cerca de um século depois., ${ }^{4,6}$ Aceita-se hoje em dia que as primeiras estimativas de prevalência pontual de transtornos alimentares na população em geral provavelmente superestimaram a bulimia nervosa. Estudos posteriores (por exemplo o de Bushnell et $\mathrm{al}^{7}$ ) concordam em geral que a bulimia nervosa ocorre em cerca de $1 \%$ das mulheres jovens ocidentais e que as síndromes de transtornos alimentares parciais ou de TASOE, aplicando os critérios do DSM-IV, ${ }^{6}$ ocorrem em $2 \%$ a $5 \%$ das mulheres jovens. ${ }^{8} \mathrm{~A}$ anorexia nervosa provavelmente ocorre em menos de $0,5 \%$ dessa população e não é comum na população em geral, por exemplo, como foi constatado por Aalto-Setala et al. ${ }^{9}$

Estudos precisos sobre a incidência têm sido mais difíceis de serem realizados, mas estudos de incidência clínica e de coortes $^{7,10}$ confirmam a existência de um aumento na incidência da bulimia nervosa desde seu reconhecimento nas últimas décadas do século passado. Pesquisas populacionais seqüenciais têm-se mostrado problemáticas e variáveis com relação à definição e seleção de casos, mas aquelas que foram realizadas não relataram um aumento desde o final dos anos $80^{11,12}$ (Hay, 2001, submetido). Os estudos sobre a incidência clínica de anorexia nervosa identificaram casos prováveis, principalmente por meio de prontuários médicos dos pacientes que se apresentam com suas complicações físicas, como infertilidade e perda de peso inexplicada. ${ }^{13,14}$ Uma revisão sistemática de 12 estudos de incidência cumulativa relatou uma incidência média anual na população em geral de 18,5 por $100.000(\mathrm{DP}=21,01)$ entre mulheres e de 2,25 por 100.000 $(\mathrm{DP}=2,63)$ por ano entre homens. ${ }^{15}$ Há evidências limitadas de alterações na incidência geral da anorexia nervosa no decorrer do tempo, ainda que Pawluck \& Gorey ${ }^{15}$ tenham relatado um aumento significativo na incidência da síndrome entre mulheres jovens de 1950 e 1992. A incidência estimada da bulimia nervosa foi mais elevada, sendo de 28,8 ( $\mathrm{DP}=29,7)$ entre mulheres e de $0,8(\mathrm{DP}=0,0)$ em homens por $100.000 \mathrm{a}$ cada ano.

Esses estudos recentes também questionaram algumas crenças anteriores sobre o papel e importância de fatores sociais e culturais nos transtornos alimentares. Por exemplo, Hoek ${ }^{13}$ não é o único a concluir que os transtornos alimentares não são um fenômeno puramente "ocidental". Outros estudos (por exemplo, Hay, ${ }^{8}$ 1998) apontaram para uma distribuição sócio-econômica mais ampla dos transtornos alimentares e para a presença de problemas de compulsão alimentar entre os homens.

\section{A aplicação de estudos epidemiológicos analíticos em trans- tornos alimentares}

Os estudos epidemiológicos analíticos aplicam métodos de pesquisa de área populacional a estudos sobre a etiologia dos transtornos tais como: estudos sobre fatores de risco e epidemiologia genética, carga social e econômica, utilização do sistema de saúde e pesquisas sobre história natural e desfecho, bem como estudos de classificação. O espaço reduzido nos impede de discutir todos eles neste artigo, que está mais focado nos estudos caso-controle recentes sobre fatores de risco, classificação e história natural e no desenvolvimento adicional da sobrecarga econômica e social e da utilização do sistema de saúde a partir de estudos de base comunitária na área de transtornos alimentares.

Esta é uma revisão seletiva com base em estudos previamente identificados e em uma pesquisa na Medline entre janeiro de 1995 e março de 2002, utilizando os termos de pesquisa "epidemiologia" e "transtornos alimentares". Cento e sessenta e um estudos foram identificados, dos quais cerca de metade eram relevantes para a área.

\section{Estudos caso-controle e prospectivos na pesquisa sobre fato- res de risco}

Os mais abrangentes estudos comunitários de caso-controle sobre fatores de risco são os descritos por Fairburn et al $^{16,18} \mathrm{Os}$ pontos fortes desses estudos são que eles analisam uma variedade de fatores de risco em diversos domínios com uma avaliação do diagnóstico baseado em entrevistas. Os principais achados foram publicados em uma série de três artigos sobre 102 mulheres com bulimia nervosa, 67 mulheres com história de anorexia nervosa, 52 mulheres com transtorno de compulsão alimentar periódica, 204 "indíviduos-controle saudáveis" e 102 mulheres com outros transtornos psiquiátricos, em sua maioria com depressão, todas com idades entre 16 e 35 anos. Os diferentes grupos comparativos permitiram a testagem da especificidade dos fatores de risco. $\mathrm{Na}$ Tabela estão sintetizados os principais achados a partir das análises de regressão multivariada setpwise, avaliando a importância das relações entre cada condição de caso e a exposição aos fatores dentro dos três domínios de fatores de risco ambientais, pessoais e da dieta. Um fator não incluído nos três domínios, a menarca precoce, surgiu também como um fator de risco específico para a bulimia nervosa. Como se pode observar na Tabela 1, alguns fatores de risco para transtornos alimentares, por exemplo, certos problemas com os pais, parecem ser específicos para a bulimia nervosa, e outros fatores como histórico de abuso sexual emergem como fatores de risco não-específicos tanto para transtornos alimentares como para outros transtornos psiquiátricos. Alguns fatores de risco foram compartilhados entre três transtornos, em geral em um continuum, de modo que fatores de risco para transtornos de compulsão alimentar periódica foram mais fracos e circunscritos. No entanto, o domínio da obesidade (história anterior e dietas) emergiu como um fator de risco para a bulimia nervosa. Este fator de risco foi considerado menor para o transtorno de compulsão alimentar periódica e inexistente para a anorexia nervosa. Este achado inesperado foi reforçado por outra pesquisa recente. ${ }^{19}$ 
Os autores reconhecem as limitações dos seus estudos, em particular pelo fato de os indivíduos com anorexia nervosa não terem sido majoritariamente recrutados na comunidade, mas de clínicas de tratamento. Esse é um problema recorrente na pesquisa populacional de transtornos alimentares e se deve, possivelmente, a pelo menos dois fatores, a saber, a relutância das pessoas com anorexia nervosa em participarem de pesquisas ${ }^{20}$ e a baixa prevalência do transtorno na população em geral como descrito acima. Além disso, os estudos de Fairburn et al, não eram cegos ao estado dos casos no momento da realização das entrevistas e os dados estavam baseados numa rememoração retrospectiva dos eventos e, portanto, sujeitos a viés.

Estudos prospectivos são raros e poucos são suficientemente grandes para detectar mais que um pequeno número de casos clínicos de transtornos alimentares que surgem à medida que a coorte envelhece. Por exemplo, o estudo de Patton et $\mathrm{al}^{21}$ somente identificou 33 novos casos femininos e oito masculinos de um transtorno alimentar, todos TASOE do tipo bulímico, ao longo de um período de dois anos e a partir de uma amostra inicial com 1947 adolescentes entre 14 e 15 anos de idade. No entanto, o recente trabalho de Johnson et al, ${ }^{22}$, a partir de um estudo longitudinal comunitário com mães, feito entre 1975 e 1993 e com crianças entrevistadas entre 1983 e 1993, reforça a importância de um exame cuidadoso adicional sobre a relação dos fatores ambientais como pouco afeto dos genitores, o que pode ser remediado através de intervenções de prevenção primária em transtornos alimentares.

\section{Estudos classificatórios e de história natural}

As pesquisas comunitárias fornecem uma importante oportunidade para estudar a classificação dos transtornos alimenta- res na medida em que eles ocorrem em indivíduos não-expostos aos vieses inerentes aos estudos com populações clínicas. A pesquisa sobre classificação diagnóstica incluiu: a análise de clusters e outros métodos estatísticos para derivar tipologias empiricamente a partir de amostras de indivíduos da comunidade com sintomas de transtornos alimentares; estudos sobre a validade de constructo dos critérios diagnósticos atuais para transtornos alimentares e, estudos sobre história natural e desfecho, as chamadas validades preditivas, de tipologias empiricamente derivadas e do sistema atual. A análise de classe latente (latent class analysis) e a análise de clusters (cluster analysis) feitas por Sullivan et $\mathrm{al}^{23}$ e Hay et $\mathrm{al}^{24}$ corroboram parcialmente os critérios diagnósticos existentes, mas também apóiam a ampliação dos critérios atuais e a existência de um espectro de transtornos.

Além disso, houve uma série de estudos de valor preditivo e de validade de constructo baseados na comunidade que compararam indivíduos a partir dos grupos diagnósticos do DSM-IV. ${ }^{6}$ Garfinkel et al, ${ }^{25}$ por exemplo, também identificaram fatores que eram mais prováveis em indivíduos com bulimia nervosa com "síndrome completa", como transtorno afetivo nos pais e alcoolismo, o que reforça o conceito de um espectro de transtornos com fatores de risco compartilhados. Striegel-Moore et a ${ }^{26}$ descobriram que mulheres da comunidade com transtorno de compulsão alimentar periódica diferiam em um certo número de parâmetros (como serem mais velhas e terem tido menos anorexia nervosa) daquelas com bulimia nervosa purgativa. Hay \& Fairburn $^{27}$ não constataram diferenças entre os transtornos de acordo com esses parâmetros, mas isso pode ter decorrido em parte de uma definição mais ampla do transtorno de compulsão alimentar periódica utilizada no último estudo. Ambos os estu-

Tabela 1 - Visão geral dos resultados de estudos caso-controle comunitários sobre os fatores de risco para transtornos alimentares realizados por Fairburn et al. ${ }^{17-19}$

Domínios de Fatores de Risco
Vulnerabilidade pessoal* $^{*}$ Ambiental**

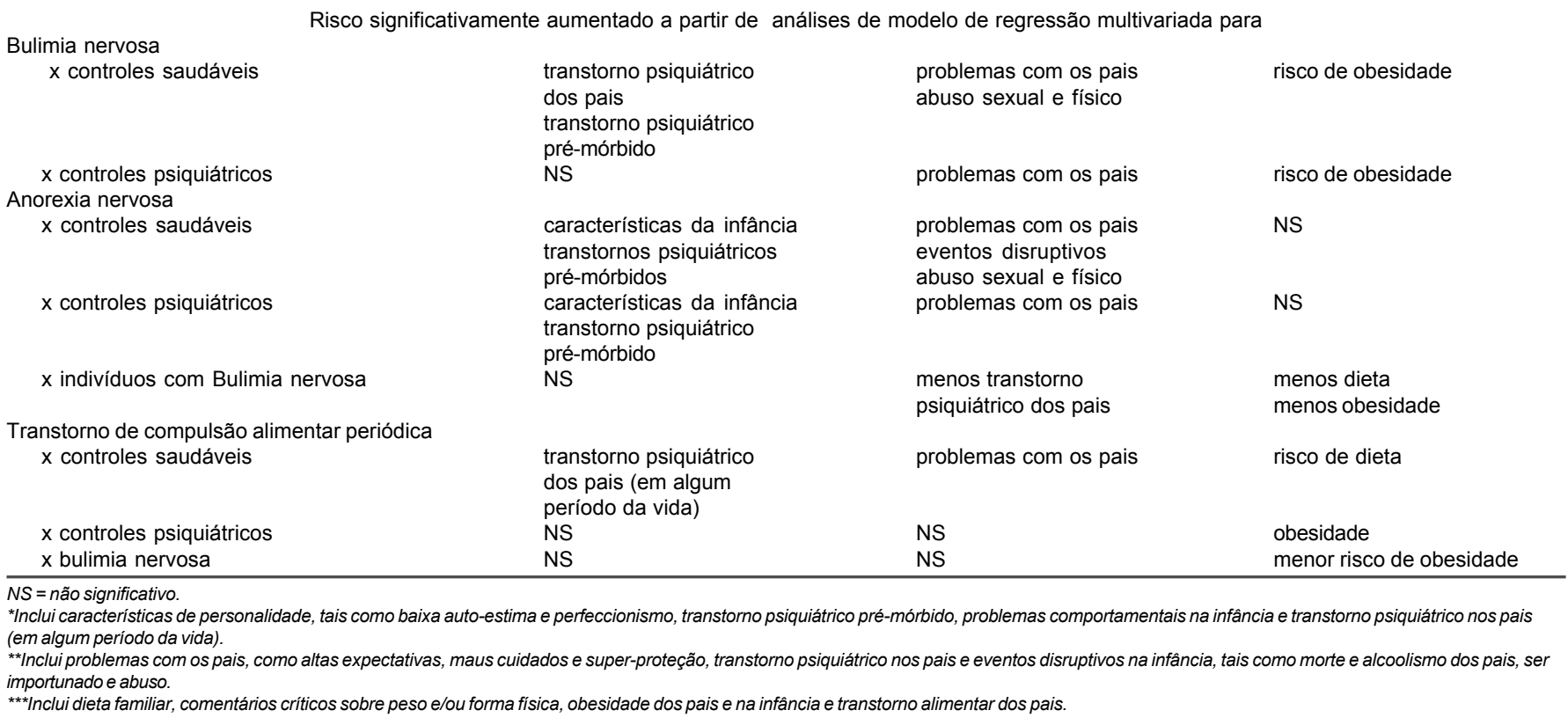


dos consideraram problemático identificar em comunidades um número suficiente de indivíduos com bulimia nervosa "não-purgativa" para tais investigações. Outros estudos examinaram questões nosológicas específicas, como o de Garfinkel et $\mathrm{al}^{25}$, que não apoiou o limiar determinado pelo DSM-IV de dois episódios de compulsão alimentar por semana, encontrando pequena diferença entre aqueles indivíduos que tinham uma freqüência de um episódio semanal em relação aos que tinham dois episódios.

Estudos de seguimento sobre a validade preditiva de diferentes sub-grupos são raros, mas Fairburn et $\mathrm{al}^{28}$ realizaram um estudo sobre o curso natural da bulimia nervosa e do transtorno de compulsão alimentar periódica durante cinco anos. ${ }^{28}$ Esse estudo encontrou que os indivíduos com bulimia nervosa tinham maior probabilidade de manter um diagnóstico de transtorno alimentar após cinco anos $(n=47,51 \%)$ comparados àqueles com transtorno de compulsão alimentar periódica $(n=7,18 \%)$. Esse desfecho diferenciado confirma o esquema classificatório que distingue esses dois transtornos alimentares. Um pequeno estudo prospectivo $(n=51)$ com base em uma amostra recrutada a partir do atendimento primário também confirmou esse achado. ${ }^{29}$ Estudos comunitários adicionais sobre a nosologia dos transtornos alimentares serão importantes para informar e confirmar ou não esses achados, mantendo e/ou revisando os esquemas classificatórios e os critérios diagnósticos atuais.

\section{Utilização do sistema de saúde e a "sobrecarga" dos transtor- nos alimentares}

A avaliação da morbidade e da "sobrecarga" mostrou que os transtornos psiquiátricos serão um dos principais problemas de saúde pública no século 21 . Um estudo australiano comprovou, por exemplo, que os transtornos mentais respondem por cerca de $30 \%$ da carga de doenças não fatais, ${ }^{30}$ ao passo que os transtornos alimentares tinham um nível similar à esquizofrenia entre as mulheres. Em um estudo no Sul da Austrália (Hay, 2001, submetido), comportamentos típicos de transtornos alimentares encontraram-se associados com escores de qualidade de vida significativamente mais baixos. Embora a sobrecarga possa ser significativa, estudos comunitários somente encon- traram uma pequena proporção de pacientes com bulimia nervosa em tratamento em qualquer período de tempo. ${ }^{31,32}$ As informações sobre como reduzir a morbidade por meio da prevenção secundária e da melhoria do "conhecimento sobre a saúde mental", ${ }^{33}$ ou seja, a compreensão dos transtornos alimentares e de seu tratamento na comunidade em geral, ainda estão, no entanto, na infância.

\section{Conclusões}

Há um certo número de problemas recorrentes nos estudos comunitários sobre transtornos alimentares. Em primeiro lugar está a dificuldade de recrutar um número suficiente de indivíduos com anorexia nervosa. Estudos longitudinais e outros estudos também consideraram problemática a (relativamente) baixa prevalência do transtorno e identificação de casos utilizando instrumentos de avaliação de boa qualidade. Os enfoques que têm sido e que podem vir a ser utilizados para superar esses problemas são o aumento da amostragem de indivíduos com alto risco de transtornos alimentares (por exemplo, o estudo de Bushnell et $\mathrm{al}^{7}$ ), o estudo de grupos diagnósticos mais amplos do que os definidos pelo DSM IV (como foi feito pela Price Foundation $^{34}$ em seus estudos sobre epidemiologia genética), e a combinação com estudos maiores sobre saúde em geral (como realizado por Garfinkel et $\mathrm{al}^{26}$ ).

Apesar desses problemas, os estudos sobre incidência e prevalência de transtornos alimentares alcançaram um consenso e, como regra, não confirmam uma incidência atual crescente, exceto possivelmente por um pequeno aumento de anorexia nervosa em mulheres jovens. A aplicação dos métodos da epidemiologia genética levou a uma maior compreensão sobre os fatores ambientais e genéticos, em comparação com os fatores sociais e econômicos, do risco do desenvolvimento de transtornos alimentares e auxiliaram no refinamento da nosologia desses transtornos. No futuro, a epidemiologia analítica poderá dar resposta a questões-chave sobre a natureza e os determinantes dos transtornos alimentares e ajudar a decidir o auxílio aos que mais necessitam, a fim de reduzir a morbidade dos transtornos alimentares na comunidade geral.

\section{Referências}

1. Myers JK, Weissman MM, Tischler GL, Holzer CE, Leaf PJ, Orvaschel H, et al. Six-month prevalence of psychiatric disorders in three communities. Arch Gen Psychiatry 1984;41:959-67.

2. Gull WW. Anorexia nervosa (apepsia hysterica, anorexia hysterica). Transact Clin Soc London 1874;7:22-8.

3. Lasegue C. De L'anorexia hysterique. Arch Gen Med $1873 ; 1: 385-403$.

4. Russell GFM. Bulimia nervosa: an ominous variant of anorexia nervosa. Psychol Med 1979;9:429-48.

5. American Psychiatric Association (APA). Diagnostic and statistical manual of mental disorders. 3rd ed. Washington (DC): American Psychiatric Association; 1980.

6. American Psychiatric Association (APA). Diagnostic and statistical manual of mental disorders. 4th ed. Washington (DC): American Psychiatric Association; 1994.
7. Bushnell JA, Wells JE, Hornblow AR, Oakley-Brown MA, Joyce P. Prevalence of three bulimic syndromes in the general population. Psychol Med 1990;20:671-80.

8. Hay PJ. The epidemiology of eating disorder behaviours: an Australian community-based survey. Int $J$ Eat Disord 1998;23:371-82.

9. Aalto-Setala T, Matrunnen M, Tuulio-Henriksson A, Poiloiainen $\mathrm{K}$, Lonnqvist J. One-month prevalence of depression and other DSM-IV disorders among young adults. Psychol Med 2001;31:791-801.

10. Hall A, Hay PJ. Eating disorder patient referrals from a population region 1977-1986. Psychol Med 1991;21:697-701.

11. Westenhoefer J. Prevalence of eating disorders and weight control practices in Germany in 1990 and 1997. Int J Eat Disord 2001;29:477-81 
12. Soundy TJ, Lucas AR, Suman VJ, Melton LJ $3^{\text {rd }}$. Bulimia nervosa in Rochester, Minnesota from 1980 to 1990. Psychol Med 1995;25:1065-71.

13. Hoek HH, van Harten PN, van Hoeken D, Susser E. Lack of relation between culture and anorexia nervosa: results of an incidence study on Curacao. N Engl J Med 1998;38:1231-2.

14. Lucas AR, Beard CM, O'Fallon WM, Kurland LT. 50-Year trends in the incidence of anorexia nervosa in Rochester, Minn.: a population-based study. Am J Psychiatry 1991;148:917-22.

15. Pawluck DE, Gorey KM. Secular trends in the incidence of anorexia nervosa: integrative review of population-based studies. Int J Eat Disord 1998;23:347-52.

16. Fairburn CG, Welch SL, Doll HA, Davies BA, O'Connor ME. Risk factors for bulimia nervosa: a community-based case-control study. Arch Gen Psychiatry 1997;54:509-17.

17. Fairburn CG, Cooper Z, Doll HA, Welch SL. Risk factors for anorexia nervosa: a community-based case-control study. Arch Gen Psychiatry 1999;56:468-76.

18. Fairburn CG, Doll HA, Welch SL, Hay PJ, Davies BA, O'Connor ME. Risk factors for binge eating disorder: a community-based case-control study. Arch Gen Psychiatry 1998;55:425-32.

19. Karwautz A, Rabe-Hesketh S, Hu X, Zhao J, Sham P, Collier DA, Treasure J. Individual-specific risk factors for anorexia nervosa: a pilot study using a discordant sister-pair design. Psychol Med 2001;31:317-29.

20. Beglin SJ, Fairburn CG. Women who choose not to participate in surveys on eating disorders. Int J Eat Disord 1992;12:113-6.

21. Patton GC, Selzer R, Coffey C, Carlin JB, Wolfe R. Onset of adolescent eating disorders: population based cohort study ofer 3 years. BMJ 1999;318:765-8.

22. Johnson JG, Cohen P, Kasen S, Brook JS. Childhood adversities associated with risk for eating disorders or weight problems during adolescence or early adulthood. Am J Psychiatry 2002;159:394-400.

23. Sullivan PF, Bulik CM, Kendler KS. The epidemiology and classification of bulimia nervosa. Psychol Med 1998;28:599-610.
24. Hay PJ, Fairburn CG, Doll H. The classification of bulimic eating disorders: a community based study. Psychol Med 1996:26;810-2.

25. Garfinkel PE, Lin E, Goering P, Spegg C, Goldbloom DS, Kennedy $\mathrm{S}$ et al. Bulimia nervosa in a Canadian community sample: prevalence and comparison of subgroups. Am J Psychiatry 1995; 152:1052-8.

26. Striegel-Moore RH, Cachelin FM, Dohm F-A, Pike KM, Wilfley DE, Fairburn CG. Comparison of binge eating disorder and bulimia nervosa in a community sample. Int J Eat Disord 2001:29:157-65.

27. Hay PJ, Fairburn CG. The validity of the DSM-IV scheme for classifying bulimic eating disorders. Int J Eat Disord 1998;23:7-15.

28. Fairburn CG, Cooper Z, Doll HS, Norman P, O'Connor M. The natural course of bulimia nervosa and binge eating disorder in young women. Arch Gen Psychiatry 2000;57:659-65.

29. Siklich I. Eating disorders in primary care: a four-year outcome study of eating disorder and sub-threshold cases [Dissertation]. Melbourne (Vic): Royal Australian and New Zealand College of Psychiatrists; 1999.

30. Mathers CD, Vos Theo E, Stevenson CE, Begg SJ. The Australian burden of disease study: measuring the loss of health from diseases, injuries and risk factors. Med J Aust 2000;172:592-6.

31. Welch SL, Fairburn CG. Sexual abuse and bulimia nervosa: three integrated case control comparisons. Am J Psychiatry 1994;151:402-7.

32. Flament M, Ledoux S, Jeammet P, Choquet M, Simon Y. A population study of bulimia nervosa and subclinical eating disorders. In: Steinhausen H, editor. Eating disorders in adolescence. Berlin: de Gruyte; 1995. p. 21-36.

33. Jorm AE. Mental health literarcy. Public knowledge and beliefs about mental disorders. Br J Psychiatry 2000;177:396-401.

34. Price Foundation Collaborative Group. Deriving behavioural phenotypes in an international, multi-centre study of eating disorders. Psychol Med 2001;31:635-45. 